It is also important to note that the proapoptotic $\mathrm{Vpr}$ protein is not the only viral mitochondrion-targeted protein to be identified as a virulence factor (9). It will be interesting to learn which other viruses produce similar mitochondrion-targeted, apoptosisregulatory, disease-relevant proteins, all of which could constitute promising pharmacological targets.

\section{Acknowledgments}

Our work is supported by grants from the Centre National de la Recherche Scientifique, the Agence Nationale pour la Recherche sur le SIDA, the Association pour la Recherche sur le Cancer, the Fondation pour la Re- cherche Médicale, the European Commission (QLG1-CT-1999-00739), the Ligue Nationale contre le Cancer, and the Ministry of Science. The authors thank Aurélien Deniaud for assistance in molecular modeling.

1. Lum, J.J., et al. 2003. $\operatorname{Vpr} \mathrm{R} 77 \mathrm{Q}$ is associated with long-term nonprogressive HIV infection and impaired induction of apoptosis. J. Clin. Invest 111:1547-1554. doi:10.1172/JCI200316233.

2. Jacotot, E., et al. 2000. The HIV-1 viral protein R induces apoptosis via a direct effect on the mitochondrial permeability transition pore. J. Exp. Med. 191:33-46.

3. Jacotot, E., et al. 2001. Control of mitochondrial membrane permeabilization by adenine nucleotide translocator interacting with $\mathrm{HIV}-1$ viral protein $\mathrm{R}$ and Bcl-2. J. Exp. Med. 193:509-520.

4. Zhang, L., Huang, Y., Yuan, H., Tuttleton, S., and Ho, D. 1997. Genetic characterization of vif, vpr, and vpu sequences from long-term survivors of human immunodeficiency virus type 1 infection Virology. 228:340-349.

5. Morellet, N., Bouaziz, S., Petitjean, P., and Roques, B. 2003. NMR structure of the HIV-1 regulatory protein VPR. J. Mol. Biol. 327:215-227.

6. Zacharias, N., and Dougherty, D. 2002. Cation- $\pi$ interactions in ligand recognition and catalysis. Trends Pharmacol. Sci. 23:281-287.

7. Sawaya, B., et al. 2000. Transdominant activity of human immunodeficiency virus type $1 \mathrm{Vpr}$ with a mutation at residue R73. J. Virol. 74:4877-4881.

8. Phenix, B., Lum, J., Nie, Z., Sanchez-Dardon, J., and Badley, A. 2001. Antiapoptotic mechanism of HIV protease inhibitors: preventing mitochondrial transmembrane potential loss. Blood. 98:1078-1085.

9. Castedo, M., Perfettini, J.L., and Kroemer, G. 2002. Mitochondrial apoptosis and the peripher al benzodiazepine receptor: a novel target for viral and pharmacological manipulation. J. Exp. Med. 196:1121-1125

10. Sali, A., and Blundell, T. 1993. Comparative protein modelling by satisfaction of spatial restraints. J. Mol. Biol. 234:779-815.

\title{
A matter of life and death: cardiac myocyte apoptosis and regeneration
}

\section{Bernardo Nadal-Ginard, Jan Kajstura, Piero Anversa, and Annarosa Leri}

Cardiovascular Research Institute, Department of Medicine, New York Medical College, Valhalla, New York, USA

J. Clin. Invest. 111:1457-1459 (2003). doi:10.1172/JCI200318611.

Of late, life has become more complicated in cardiovascular biology. Life was simpler when the prevalent dogma stating that the heart is a terminally differentiated organ without regenerative capacity remained unchallenged (1). This static view of the myocardium implied that both myocyte death and myocyte replication played no meaningful role in cardiac homeostasis and could be safely ignored. In the absence of myocyte renewal, cell death by apoptosis or necrosis had to be extremely low or non-existent to explain the preservation of the cardiac mass throughout the lifespan of the individual. Even very low

\footnotetext{
Address correspondence to: Bernardo NadalGinard, Cardiovascular Research Institute, Department of Medicine, New York Medical College, Valhalla, New York 10595, USA. Phone: (914) 594-4755; Fax: (914) 594-4406;

E-mail: b_nadal-ginard@nymc.edu.

Conflict of interest: The authors have

declared that no conflict of interest exists.

Nonstandard abbreviations used:

mammalian sterile 2-like kinase 1 (Mst1).
}

rates of myocyte death would have resulted in the complete disappearance of the myocardium in a few decades. Thus, it is not surprising that the existence of myocyte apoptosis remained controversial during the past decade (2). Recent findings indicating the presence in the adult myocardium of a cell population with the behavior and potential of cardiac stem cells has challenged the status quo, provided an explanation for the existence of a subpopulation of immature cycling myocytes and embraced myocyte death and myocyte renewal as the two sides of the proverbial coin of cardiac homeostasis (3). This myocyte renewal depends on the differentiation of primitive cells into immature myocytes that might divide two to four times before becoming terminally differentiated and permanently withdrawn from the cell cycle. There is no evidence that already mature cardiomyocytes can de-differentiate, reenter the cell cycle, and proliferate. In this new light, the presence, regulation, and physiological consequences of myocyte apoptosis have gained new significance. Two papers in this issue of the JCI highlight the effect of this type of myocyte death in cardiac performance and provide new insights on the role of myocyte death and renewal in cardiovascular physiology $(4,5)$.

\section{Diffuse cell death leads to dilated cardiomyopathy}

Although it is well accepted that myocyte death is a determining factor of ventricular dysfunction and end-stage failure (6), whether diffuse myocyte apoptosis can, by itself, cause cardiac failure has remained controversial. The pattern of cell death within the myocardium has distinct consequences on cardiac function. Moderate scattered myocyte death has a greater negative effect on ventricular hemodynamics than equivalent segmental myocyte loss (7). It takes the destruction of $40-50 \%$ of left ventricular myocytes after coronary artery occlusion to produce cardiac failure (8), while a $10-20 \%$ myocyte dropout dispersed throughout produces the same result (7). The two animal models of myocyte apoptosis published in this issue of the JCI $(4,5)$ provide conclusive proof that myocyte dropout by itself can cause cardiac failure. In these studies, appearance of dilated cardiomyopathy is mediated by diffuse myocyte apoptosis across the 

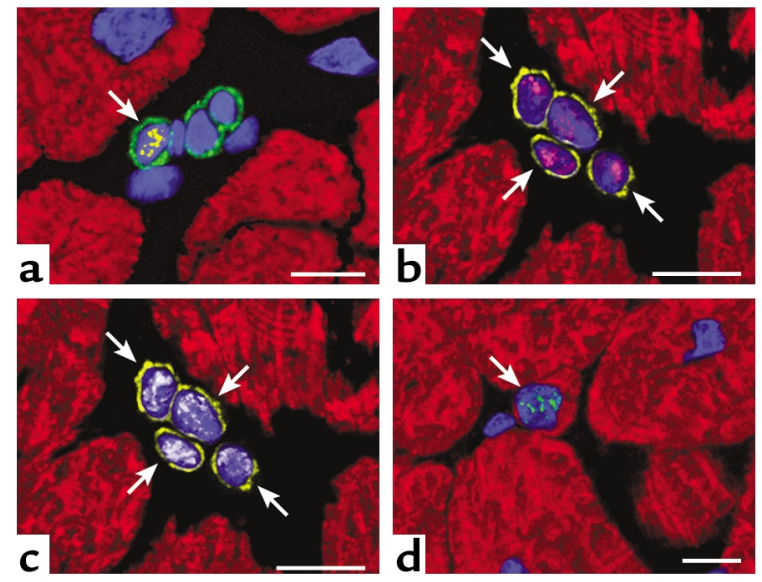

\section{Figure 1}

Developing myocytes in a pathologic human heart. (a) Cardiac primitive cells expressing $c$-kit on the surface membrane (green fluorescence). Nuclei are stained by propidium iodide (blue fluorescence). The nucleus of one of these c-kit-positive cells is labeled by the marker of cell proliferation Ki67 (yellow fluorescence; arrow). (b and c) Four myocyte progenitors illustrated in the same field. They are recognized by the presence of the stem cell surface antigen multi drug resistance protein 1 (MDR-1) (yellow fluorescence; arrows) and by the nuclear localization of the myocyte specific transcription factor myocyte enhancer factor 2 (MEF2) (b, magenta fluorescence). These early committed cells are cycling as documented by the expression of mini chromosome maintenance protein 5 (MCM5) (c, white fluorescence). MCM5 is another marker of the cell cycle. (d) An amplifying myocyte (arrow), which has lost the stem cell surface antigens, is shown. However the nucleus (blue fluorescence) expresses telomerase (green fluorescent dots). A thin layer of myocyte cytoplasm is recognized by the red fluorescence of cardiac myosin heavy chain. Confocal microscopy; scale bars $=10 \mu \mathrm{m}$.

ventricular wall. The effector pathway implicated in this myocyte death involves the activation of caspases. In the study from Kitsis' laboratory, a direct activation of caspase 8 was produced by regulated overexpression of a constitutively active form of this protein in myocytes (5). In the report from Sadoshima's laboratory, caspase function was stimulated indirectly by cardiac-specific overexpression of mammalian sterile 2-like kinase 1 (Mst1), a substrate and activator of caspase 3 (4). These two transgenic animals might provide useful models for the long-term decompensated human heart in which an imbalance between myocyte death and regeneration favors cardiac dilation and failure $(9,10)$.

Many transgenic animal models of dilated cardiomyopathy have been made in an attempt to mimic the anatomical, physiological, and clinical features of human dilated cardiomyopathy (11-13). However, in most of these models ventricular dysfunction has been attributed to depressed myocyte contractility. Cell death was not studied and, therefore, whether myocyte cell loss participated in the initiation and evolution of heart failure remains to be established. Although in the final assessment, there is no evidence indicating that both myocyte loss and reduced contractility of the remaining myocytes are always present in heart failure.

Overexpression of tropomodulin, an actin filament regulatory protein, results in scattered cell death, apoptotic and necrotic in nature, which is a crucial determinant of the dilated failing heart (14) and can be rescued by overexpression in myocytes of IGF1 (14). Ablation of telomerase results in telomeric shortening and activation of p53 (15) leading to a diffuse pattern of myocyte apoptosis, ventricular dilation, and cardiac failure. Finally, pacing-induced heart failure in dogs is characterized by massive loss of myocytes and cavitary dilation (16). Importantly, caspase activation modulates myocyte apoptosis in ventricular pacing (17). There seems to be a great deal of similarity between the biochemical events triggering myocyte death with pacing in dogs (17) and that observed following caspase 8 (5) and Mst1 (4) overexpression in transgenic mice.

\section{High myocyte death implies myocyte regeneration}

The elegance of the two transgenic mouse models of dilated cardiomyopathy published here $(4,5)$ is that the cardiac pathology depends strictly on myocyte death as clearly demonstrated by rescue of the phenotype by inhibition of caspase activation. Apoptotic cell death in these animals produces changes in heart size and shape that are consistent with an architectural rearrangement of myocytes, involving sideto-side slippage of cells within the wall (18). As suggested (4), such a reorganization of the myocyte compartment could account for the increase in cavitary volume and reduction in wall thickness in these transgenic mice. This condition results in abnormal levels of resting tension with activation of the cell death pathway and a further reduction in myocardial performance, thus establishing a vicious feedback loop. Not surprisingly, inhibition of myocyte death in heart failure attenuates ventricular dilation, reactive myocyte hypertrophy and diastolic stress, demonstrating unequivocally the crucial role of cell death in pathologic remodeling $(14,19,20)$.

The levels of apoptosis measured by Sadoshima and colleagues (4) and Kitsis and colleagues (5), which are similar to those measured in the decompensated human heart of ischemic and non-ischemic origin (6), at first glance might seem low and raise questions about their relevance to the pathogenesis of heart failure. To evaluate their significance it is illuminating to compute the resulting myocyte loss over time. Unfortunately, an essential parameter for this computation, the time required for completion of apoptosis in vivo, is unknown. In vitro apoptosis is completed in approximately 2 hours (21). A conservative estimate for this process in vivo might be 4 hours. On these bases, nearly $1.8 \%$ of myocytes should die per day in the Mst 1 mouse. If this were the case, a rapid loss of cardiac mass would occur and the halflife of the heart would be about 38 
days. The extent of apoptosis in the FK506-binding protein-caspase 8 mouse is lower and consequently the decline in myocardial mass would occur in a longer period of time.

The inevitable conclusion from these indirect measurements is that cell death cannot be the only cellular mechanism implicated in the adaptation of the heart to overexpression of caspase 8 or Mst1 in myocytes. That despite the remarkable level of myocyte death and in the absence of myocyte hypertrophy, cardiac weight was comparable in wildtype and transgenic mice (4) further reinforces this view. The obvious alternative for the heart to maintain its mass and exert its function dictates that new myocytes had to be formed to replace those continuously lost. Unfortunately, myocyte regeneration was not measured, but it offers the only explanation for the cardiac phenotypes described. This conclusion is supported by the observation that in animals and humans the normal heart undergoes a continuous myocyte turnover, which increases under stressful situations (3, 22). Perhaps, in absence of Mst1, the adaptive response to work-overload is hyperplasia instead of hypertrophy.

Recently, a better understanding has been developing about the source of new and replicating myocytes in the adult heart. Undifferentiated cells expressing surface antigens commonly found in bone marrow progenitor cells are present in the atria and ventricle of the human and rodent heart $(3,22,23)$. These cells differentiate in the various cell lineages of the adult heart $(3,22,23)$ and can be found at all stages of differentiation, ranging from the most primitive to small immature and cycling myocytes. Importantly, these undifferentiated and early committed cells express cardiac and myocyte specific transcription factors and are positive for cell cycle markers and telomerase (Figure 1). Thus, although most cardiac myocytes permanently withdraw from the cell cycle, the heart has regenerative potential and it is not a terminally differentiated organ.

In conclusion, the studies from Kitsis' and Sadoshima's laboratories demonstrate that myocyte death can be a major component of the decompensated failing heart. Although not analyzed directly, the phenotype and even the reduced survival of these animals can only be explained by concomitant myocyte regeneration. Taken together with other available data, the results presented in these two papers make a strong case for the need to develop a new understanding of normal and pathological cardiac homeostasis in which both myocyte death and myocyte renewal are essential for the maintenance of cardiac function and its adaptation to different physiological and pathological demands $(3,6,22-24)$.

1. MacLellan, W.R., and Schneider, M.D. 2000 Genetic dissection of cardiac growth control pathways. Annu. Rev. Physiol. 62:289-319.

2. Kang, P.M., and Izumo, S. 2000. Apoptosis and heart failure: a critical review of the literature. Circ. Res. 86:1107-1113.

3. Nadal-Ginard, B., Kajstura, J., Leri, A., and Anversa, P. 2003. Myocyte death, growth, and regeneration in cardiac hypertrophy and failure. Circ. Res. 92:139-150.

4. Yamamoto, S., et al. 2003. Activation of Mst1 causes dilated cardiomyopathy by stimulating apoptosis without compensatory ventricular myocyte hypertrophy. J. Clin. Invest. 111:1463-1474. doi:10.1172/JCI200317459.

5. Wencker, D., et al. 2003. A mechanistic role for cardiac myocyte apoptosis in heart failure. J. Clin. Invest. 111:1497-1504. doi:10.1172/JCI200317664.

6. Olivetti, G., et al. 1997. Apoptosis in the failing human heart. N. Engl. J. Med. 336:1131-1141.

7. Anversa, P., Zhang, X., Li, P., and Capasso, J.M. 1992. Chronic coronary artery constriction leads to moderate myocyte loss and left ventricular dysfunction and failure in rats. J. Clin. Invest. 89:618-629.

8. Anversa, P., Olivetti, G., Meggs, L.G., Sonnenblick, E.H., and Capasso, J.M. 1993. Cardiac anatomy and ventricular loading after myocardial infarction. Circulation. 87:22-26.

9. Kajstura, J., et al. 1998. Myocyte proliferation in end-stage cardiac failure in humans. Proc. Natl. Acad. Sci. U. S. A. 95:8801-8805.

10. Guerra, S., et al. 1999. Myocyte death in the failing human heart is gender dependent. Circ. Res. 85:856-866.

11. Nicol, R.L., Frey, N., and Olson, E.N. 2000. From the sarcomere to the nucleus: role of genetics and signaling in structural heart disease. Annu. Rev. Genomics Hum. Genet. 1:179-223.

12. Ikeda, Y., and Ross, J., Jr. 2000. Models of dilated cardiomyopathy in the mouse and the hamster. Curr. Opin. Cardiol. 15:197-201.

13. Wallace, D.C. 2002. Animal models for mitochondrial disease. Methods Mol. Biol. 197:3-54.

14. Welch, S., et al. 2002. Cardiac-specific IGF-1 expression attenuates dilated cardiomyopathy in tropomodulin-overexpressing transgenic mice. Circ. Res. 90:641-648.

15. Leri, A., et al. 2003. Ablation of telomerase and telomere loss leads to cardiac dilatation and heart failure associated with $\mathrm{p} 53$ upregulation. $E M B O$. J. 22:131-139.

16. Kajstura, J., et al. 1995. The cellular basis of pacing-induced dilated cardiomyopathy: myocyte cell loss and myocyte cellular reactive hypertrophy. Circulation. 92:2306-2317.

17. Cesselli, D., et al. 2001. Oxidative stress-mediated cardiac cell death is a major determinant of ventricular dysfunction and failure in dog dilated cardiomyopathy. Circ. Res. 89:279-286.

18. Olivetti, G., Capasso, J.M., Sonnenblick, E.H., and Anversa, P. 1990. Side-to-side slippage of myocytes participates in ventricular remodeling acutely after myocardial infarction in rats. Circ. Res. 67:23-34.

19. Li, Q., et al. 1997. Overexpression of insulin-like growth factor- 1 in mice protects from myocyte death after infarction, attenuating ventricular dilation, wall stress, and cardiac hypertrophy. J. Clin. Invest. 100:1991-1999.

20. Li, B., et al. 1999. IGF-1 attenuates the detrimental impact of nonocclusive coronary artery constriction on the heart. Circ. Res. 84:1007-1019.

21. Oberhammer, F.A., et al. 1992. Induction of apoptosis in cultured hepatocytes and in regressing liver by transforming growth factor $\beta 1$. Proc. Natl. Acad. Sci. U. S. A. 89:5408-5412.

22. Anversa, P., and Nadal-Ginard, B. 2002. Myocyte renewal and ventricular remodeling. Nature. 415:240-243.

23. Quaini, F., et al. Chimerism of the transplanted heart. N. Engl. J. Med. 346:5-15.

24. Beltrami, A.P., et al. 2001. Evidence that human cardiac myocytes divide after infarction. N. Engl. J. Med. 344:1750-1757. 